\title{
La novela histórica como herramienta interdisciplinar en los Estudios Generales ${ }^{1}$
}

\section{The historical novel as an interdisciplinary tool in General Studies}

Recibido: 3 de octubre de 2021 | Aprobado: 22 de noviembre de 2021

\section{Resumen}

Por su naturaleza la novela histórica constituye una herramienta interdisciplinar, puesto que en ella converge un conjunto de saberes que tocan de cerca distintas áreas del conocimiento y como tal, juega un papel importantísimo en los Estudios Generales. Esta propuesta se circunscribe específicamente en el ámbito de los estudios Generales tomando en cuenta que desde ellos se promueve "la inter y la transdisciplinariedad". El objetivo que se persigue a través de este estudio es promover la novela histórica como herramienta interdisciplinar para la articulación de diferentes áreas del conocimiento. El carácter expositivo y argumentativo de este trabajo parte de la investigación, lectura, análisis y consulta de diferentes fuentes bibliográficas que corroboran el valor educativo de la literatura como escenario del que forma parte la novela histórica, así como de los beneficios específicos que ofrece trabajar con este género, por lo que este ensayo se apoya en la ejemplificación y explicitación de los saberes implícitos en la novela histórica a partir de una en específico: El reino de este mundo de Alejo Carpentier. Para valorar el impacto de haber trabajado esta novela en el aula, se tomó en cuenta la implementación de actividades en las que se articulan todos los saberes implícitos en ella y un conjunto de instrumentos para la sistematización del progreso de los estudiantes en relación con las aspiraciones de un enfoque interdisciplinar. Dentro de los instrumentos de evaluación se privilegiaron las rúbricas, escalas estimativas y listas de cotejo. En síntesis, este artículo permitirá acercarnos a la novela histórica y a la literatura desde una perspectiva diferente: recursos a través de los que se puede articular conocimientos desde las distintas áreas convergentes en ellas.

Palabras clave: novela histórica, interdisciplinar, Estudios Generales, literatura, Carpentier

1 Ponencia presentada en el XII Simposio Internacional de Estudios Generales (modalidad virtual), Pontificia Universidad Católica Madre y Maestra (PUCMM), República Dominicana, y Red Internacional de Estudios Generales (RIDEG).

* Doctora en Estudios del Español, Lingüística y Literatura por la Pontificia Universidad Católica Madre y Maestra. Docente por asignatura de la Pontificia Universidad Católica Madre y Maestra. Para contactar a la autora: gm.caba@ce.pucmm.edu.do

ISSN (en línea): 1814-4152 / Sitio web: http://cuaderno.pucmm.edu.do CÓMO CITAR: Caba Liriano, G. (2022). La novela histórica como herramienta interdisciplinar en los Estudios Generales. Cuaderno de Pedagogía Universitaria, 19 (37), $75-82$. 


\section{Abstract}

By its nature, the historical novel constitutes an interdisciplinary tool, since it converges a set of knowledge that closely touches different areas of knowledge, and as such plays a very important role in General Studies. This proposal is specifically circumscribed in the field of General Studies, taking into account that from them "inter and transdisciplinarity" are promoted. Due to its educational potential, the historical novel as an interdisciplinary tool plays an important role in the construction of citizenship and democracy, which is an aspiration of General Studies. The objective pursued through this work is: To highlight the value of the historical novel as an interdisciplinary tool for the articulation of different areas of knowledge. The expository and argumentative nature of this work is based on the research, reading, analysis and consultation of different bibliographic sources that corroborate the educational value of literature as the setting of which the historical novel is part, as well as the specific benefits that working with the historical novel relies on the exemplification and clarification of the knowledge implicit in the historical novel from a specific one: The kingdom of this world by Alejo Carpentier. In summary, this article will allow to approach the historical novel and literature from a different perspective: resources through which they can articulate knowledge from the different areas converging on them.

Keywords: historical novel; interdisciplinary; Liberal Arts; literature; Carpentier.

\section{Introducción}

Por su potencial educativo, la novela histórica como herramienta interdisciplinar juega un papel importante en la construcción de ciudadanía y democracia, lo cual es una aspiración de los Estudios Generales.

Actualmente, existen muchos estudios a nivel nacional e internacional que corroboran el valor de la novela histórica, sin embargo, en el plano nacional no hay ninguno que la promueva como estrategia, como medio para la construcción de conocimientos interdisciplinares de los Estudios Generales y es aquí donde cobra mayor importancia este trabajo de investigación.

Dentro de los estudios realizados en el contexto internacional se destaca el de Nava (2014), en el que plantea como una problemática importante la separación entre las disciplinas y concibe la novela histórica como un eje articulador que puede mediar en el acercamiento de las disciplinas. En cuanto a la problemática se refiere: "Existe una separación entre las disciplinas integrantes de los programas de estudios escolares de los distintos niveles educativos, que los profesores intentan disminuir (...)" (p. 58). En el caso específico de la novela histórica como solución, considera el subgénero como sigue: "La novela histórica considerada como arma de denuncia y crítica (...)" (p. 60). En ese orden, expone: "Los profesores de historia que quieran auxiliarse de las novelas y relatos históricos pueden hacer una clasificación operativa y convencional de estos materiales literarios con el propósito de facilitar el trabajo de los alumnos" (p. 60). Desde luego, esta propuesta articuladora no es una cuestión exclusiva de los docentes de historia como lo plantea la autora, sino también de los de Lengua Española y de Literatura.

En este mismo escenario destaca el trabajo realizado por Ruiz (2008), quien concibe la novela histórica "no solo como una diversión, sino como fuente de conocimientos de valores" y le atribuye gran importancia para la enseñanza de la historia. Una de las riquezas que proporciona la novela histórica es el poder de enfocar una misma época histórica desde diferentes vertientes, lo cual constituye para los estudiantes una variedad de opciones.

A la luz de los planteamientos anteriores, surgen las siguientes preguntas de investigación: ¿Por qué es importante promover la novela histórica como herramienta interdisciplinar para la construcción de conocimientos en los Estudios Generales? ¿Cuál es el potencial educativo implícito en las novelas históricas? Para dar respuestas a estas interrogantes, nos proponemos promover la novela histórica como herramienta en la construcción de conocimientos interdisciplinares en el ámbito de los 
Estudios Generales. Además, este estudio pretende evidenciar el contenido educativo implícito en la novela histórica como herramienta interdisciplinar.

Para la organización de este estudio desarrollaremos los siguientes tópicos: Naturaleza de los Estudios Generales, como ámbito en el que se promueve la novela histórica; la literatura como área de la que forma parte la novela histórica y como eje articulador de diferentes disciplinas en el contexto de los Estudios Generales la novela histórica como herramienta interdisciplinar en los Estudios Generales y el potencial educativo de la novela histórica, a la luz de El reino de este mundo de Alejo Carpentier. Luego ofreceremos las conclusiones a las que arribamos.

1. Naturaleza de los Estudios Generales, como ámbito en el que se promueve la novela histórica

En atención al primer tópico, es importante dar respuesta a la siguiente interrogante: ¿Por qué los Estudios Generales merecen estudios interdisciplinares? Para ello, es importante partir de algunas características de los Estudios generales. Al respecto, PUCMM (2016) refiriéndose a la razón de ser de los Estudios Generales expone lo siguiente: "promueve el análisis y la expresión, incentiva el amor por la verdad, promueve el conocimiento del contexto histórico cultural, a partir de la exploración de los vínculos entre él y la ciudadanía". Es decir, que actualmente los Estudios Generales se conciben como escenario para la formación de sujetos críticos, que además puedan vincular las teorías con su contexto, con el contexto histórico y su relación con la ciudadanía.

De igual manera, PUCMM (2016) señala que el objetivo principal de los Estudios Generales consiste en: "Promover la educación plena e integral del ser humano, mediante una visión holística, no segmentada de la realidad. Por ello, surge la iniciativa de promover el análisis de la novela histórica y la literatura en sentido general como herramientas, medios, estrategias, con el fin de corroborar su potencial en el ámbito de la educación y, de manera específica, en los Estudios Generales.
2. La literatura como área de la que forma parte la novela histórica y como eje articulador de diferentes disciplinas en el contexto de los Estudios Generales

Tal como se ha expuesto recientemente, una herramienta fundamental para la inter y transdisciplinariedad es la literatura, y teniendo en cuenta que la novela es un género fundamental y, posiblemente, uno de los más trabajados de este ámbito, se presentará, a continuación, el punto de vista de algunos autores sobre el papel de la literatura en la educación, a modo de contextualización, antes de tocar de lleno el tema de la novela histórica como herramienta interdisciplinar.

Tanto por su diversidad de géneros, como por su carácter estético, lúdico, social y cultural, la literatura juega un papel importantísimo en el ámbito educativo y de manera muy especial en los Estudios Generales por los objetivos y fines que desde ellos se persigue. Por ejemplo, refiriéndose al rol que desempeñan las artes y las humanidades y, por ende, la literatura, Nussbaum (2010) expone: “(...) las artes y las humanidades son fundamentales, tanto en la educación primaria y secundaria, como en la universidad" (p. 12). De igual manera, Reverte (2015) explica que, "La literatura da herramientas prácticas de vida, se adelanta a lo que esos jóvenes tendrán que vivir en el futuro. Les proporciona (...) armas para combatir, mecanismos para comprender. Pone a su disposición esos tres mil años de cultura (...)". Destacando así su valor y la necesidad de ponerla al alcance de los jóvenes.

A la luz de las ideas anteriores, se concibe la literatura como una herramienta a través de la cual se accede a la cultura del mundo, lo cual amplía el acervo cultural de los jóvenes y les pone en contacto con la realidad cultural global. De igual modo, además de favorecer la competencia de comprensión y a las competencias comunicativas en sentido general, contribuye de forma específica con la comprensión de la transformación cultural, la herencia cultural y los componentes propios de cada cultura.

Por tanto, la literatura encierra un conjunto de saberes que constituye un escenario ideal para desarrollar competencias y construir conocimientos de manera interdisciplinar en los estudiantes, 
potenciando sus habilidades de forma integral; por lo cual la literatura es una aliada de la educación de todos los niveles, y de manera especial, en los Estudios Generales, puesto que contribuye con el logro de sus objetivos.

De igual manera, la literatura permite que los estudiantes se sitúen en diferentes épocas desde las cuales pueden generar reflexiones críticas de ellas en sí mismas, de los personajes que protagonizan los hechos, lo que posibilita establecer comparaciones con la actualidad. A partir del análisis y reflexión de los contenidos subyacentes en los diferentes géneros, los estudiantes pueden crear mesas de diálogos orientadas hacia la reflexión de cómo afectan al colectivo social determinadas problemáticas presentes en los textos literarios aplicables a su mundo actual.

En fin, la literatura es una herramienta de apoyo para la educación, una puerta de acceso al conocimiento, un escenario para el esparcimiento y la recreación, para la generación de nuevas ideas y nuevos productos. La literatura es una fuente de conocimientos interdisciplinares, cada uno de los géneros que la conforman es una especie de dispositivo cargado de informaciones diversas que llegan al estudiante dada su forma estética, lúdica y creativa.

3. La novela histórica como herramienta interdisciplinar en los Estudios Generales

En lo que se refiere al tercer tópico, orientado a la promoción de la novela histórica como herramienta interdisciplinar en los Estudios Generales, es importante destacar que este subgénero, por su naturaleza y sus puntos de encuentro con diferentes disciplinas, facilita la construcción de conocimientos integrados y holísticos, que, como se pudo observar en los párrafos que anteceden, se manifestó que ese es el principal objetivo de los Estudios Generales.

Antes de profundizar en el desarrollo del tópico es importante situar la novela histórica como subgénero y especificar algunas características y conceptualizaciones al respecto. En este sentido, Mata (1995) define la novela histórica como subgénero narrativo, en el que convergen elementos históricos y de ficción. En tal sentido, señala: "la novela perderá el derecho de apellidarse histórica, degenerando hacia un tipo de novela con un vago fondo histórico (...)" (p.37). El mismo autor citado anteriormente es consciente de que no es fácil lograr este equilibrio, sin embargo, lo considera fundamental al momento de clasificar una novela como histórica. Este fondo histórico al que hace referencia es precisamente uno de los elementos que hacen la novela histórica una fuente rica de conocimientos que se puede explotar desde los Estudios Generales.

Respecto a la caracterización de la novela histórica, Mata (1995, p.15) especifica algunas características principales del subgénero en cuestión, tales como, situar: "su acción (ficticia, inventada) en un pasado (real, histórico) más o menos lejano". De modo que, la ficción debe contar siempre con un telón de fondo con bases en la realidad y que simultáneamente contemple el componente histórico, para que de este modo se pueda alcanzar la denominación de novela histórica. De igual forma, otra característica que le atribuye Mata (1995, p.16) a la novela histórica es "la reconstrucción de la época en que sitúa la acción". Desde luego, es importante destacar que la reconstrucción de una época implica todos sus componentes: históricos, culturales, económicos y sociales, lo cual constituye un panorama rico para diferentes disciplinas convergentes en los Estudios Generales.

En relación al valor del componente histórico, Nava (2014) muestra cómo la historia puede servirse de la literatura para desarrollar competencias y construir conocimientos a la vez que se hace más llevadero el trabajo de los alumnos por el placer estético que ofrece la literatura y el componente de ficción. Desde luego, el docente tiene el reto de acompañar a los alumnos en el proceso de separar realidad y ficción. En el caso específico de este subgénero, se convierte en un recurso, ya que es una herramienta con un alto componente histórico que puede ser explotada desde las aulas, apoyándose simultáneamente de fuentes bibliográficas históricas que les permita a los estudiantes juzgar críticamente los acontecimientos contenidos de ambas, establecer diferencias entre la plasmación ficcional o real de los hechos y formular sus propias inferencias. Por otro lado, Ruiz (2008) concibe la novela histórica como fuente de conocimientos de valores y la considera como 
un recurso imprescindible para el estudio de la historia. En este sentido, una de las riquezas que proporciona la novela histórica es el poder de enfocar una misma época histórica desde diferentes vertientes, lo cual constituye para los estudiantes una variedad de opciones. Por ejemplo, en el caso de las novelas históricas dominicanas que versan sobre la tiranía trujillista, existen novelas que permiten conocer la época desde la perspectiva del conflicto domínico - haitiano, la crueldad, la violación de los Derechos Humanos y los actos sangrientos que la caracterizan; otras, en cambio, enfocan la época desde los actos heroicos de los líderes revolucionarios y otras desde el punto de vista de "la masculinidad del Jefe", como es el caso de "La fiesta del chivo", solo para citar algunos casos. Al final, cada una conduce a la misma época, pero es ideal ofrecerle al estudiantado esta diversidad de aristas y darle la opción de elegir, lo cual constituye para ellos una oportunidad para decidir, aumentando así su motivación al sentirse involucrados en la selección de sus lecturas.

Hasta aquí, se ha presentado tanto la novela histórica, así como el ámbito en el que se inscribe (la literatura), como herramienta interdisciplinar de gran importancia para el logro de los objetivos que persiguen Los Estudios Generales, en el caso de la novela histórica, definitivamente, es un género que se constituye en una especie de dispositivo en el que convergen los siguientes saberes: políticos, sociales, lingüísticos, geográficos, culturales, artísticos, ficcionales y religiosos. Es importante ahora evidenciar a partir del análisis y la ejemplificación todos los atributos que se le han conferido con anterioridad al subgénero.

4. Potencial educativo de la novela histórica, a la luz de El reino de este mundo de Alejo Carpentier.

Antes de iniciar con el estudio aplicado a la novela El reino de este mundo, para evidenciar su potencial educativo, es importante destacar el valor que algunos autores le atribuyen a la novela histórica en sentido general, como fuente de conocimientos. En este orden, Mata (1995) señala que: "Todos sentimos una curiosidad por la historia (...) nuestro conocimiento se enriquece con otros aspectos hasta ahora descuidados: la historia económica, cultural, religiosa, (...) la vida cotidiana, o la protagonizada por las mujeres con lo que se camina hacia la denominación historia total”. (p.36). Esta reflexión que ofrece el autor citado corrobora el valor de la novela histórica como herramienta educativa para los Estudios Generales.

Para ofrecer un ejercicio interdisciplinar de la novela histórica, se expondrá la dinámica llevada a cabo en las aulas. En primer lugar, fue necesario enfocar los saberes implícitos en ellas en tres áreas: ciencias sociales y humanas, literatura y lengua española. En el ámbito de las ciencias sociales y humanas, se ubicaron los saberes: políticos, sociales, geográficos, religiosos y éticos. En el ámbito de la literatura, se ubicaron los saberes: artísticos, estéticos y ficcionales, mientras que en el ámbito de la lengua española, se ubicaron aspectos lingüísticos relacionados con lo estructural, normativo y funcional de la lengua. Es importante, a modo de contextualización, ofrecer al lector una mirada de los tres ámbitos en los que se han agrupado los saberes convergentes en la novela histórica. En el caso de las ciencias sociales y humanas, sirva para su justificación la siguiente cita: Las Ciencias Sociales (2018) se definen como "un conjunto de disciplinas que estudian fenómenos relacionados con la realidad del ser humano, la economía, la sociología, la política, la antropología, la geografía y la historia (...)" (p.1).

En lo que se refiere a la literatura, Borja Orozco et al. (2010) definen la literatura como: "un entrecruzamiento de múltiples elementos que ejercen fuertes presiones en la valoración de las obras de su circulación, de su poder en la construcción de imaginarios, de la influencia en la imaginación de los sujetos" (p.1). Por su parte, en lo que se refiere a la lengua española, se asumirá como lengua materna, como idioma nacional a través del cual nos comunicamos, haciendo uso de sus funciones comunicativas, sociales y normativas, que no hace falta definir.

Para la puesta en evidencia de los múltiples saberes que convergen en la novela histórica y que anteriormente fueron agrupados en tres tipos, se tomó como ejercicio la novela El reino de este mundo de Alejo Carpentier. Esta novela se inscribe en el ámbito de la literatura hispanoamericana, versa sobre la Revolución haitiana considerado el 
primer movimiento revolucionario de América latina. La novela se caracteriza por el predominio de lo real - maravilloso y cuenta con una gran popularidad. Por tanto, lo concerniente al poder político queda expuesto a través del uso y abuso del poder. Henry Christopher es comparado con un rey. Todos los derechos quedan adheridos a su persona, quien se proyecta como un ser que goza de todos los privilegios, oprime y maltrata a la población.

Carpentier (1972) hace posible la apreciación de la función social de la literatura, la cual queda reflejada a través de las luchas de clases, asunto que se pone en evidencia por medio de las categorías: "siervo/ amo; esclavo/amo; doméstica/ amo; señora/ negra" (p. 19)., que son la mejor representación de este apartado. De igual manera, a lo largo de toda la novela queda retratado el aspecto racial, al usar los conceptos "negro/ negra" (p. 24)., como sinónimos de inferioridad. De igual manera, el autor hace notar las formas de rechazo y exclusión social, específicamente, en la figura de "MacKandal" ( $p$. 29). quien al perder un brazo, pierde su valor como ser humano y pasa a ser considerado como inútil para el trabajo. Otros problemas de carácter social que quedan ampliamente ilustrados en el desarrollo de la novela son: "torturas físicas a los esclavos" ( $p$. 39).; "abuso sexual a las negras" (p. 42).; "violación a menores" (p. 65).; "negros asesinados de forma brutal" (p. 79).

De igual forma, los aspectos éticos versus corrupción quedan expuestos en la novela: "el gobernador dispensaba favores a cambio de mujeres" (p. 89). Los aspectos de la religiosidad ocupan también un puesto importante en la novela de Carpentier, los cuales pueden apreciarse en "la celebración de ritos religiosos" (p. 117). Igualmente, que los componentes culturales quedan evidenciados a través de "las creencias" (p. 62)

En síntesis, para concluir con el primer bloque de saberes convergentes en la novela histórica que se han ubicado en las ciencias sociales y humanas, vale la pena resaltar que la esclavitud queda representada a lo largo de toda la novela en Ti Noel, quien nunca pudo liberarse de su condición de esclavo. Una característica inherente que se le atribuye de forma satírica al esclavo es la "ignorancia" (p. 19).
Entrando al grupo de saberes ubicados en el ámbito literario, en la novela El reino de este mundo Carpentier (1972) se sirve de la ficción al máximo esplendor. Lo real maravilloso abraza de principio a fin la novela. En este contexto se presentan algunos ejemplos: "mujeres que eran violadas por felinos, personas que respondían a órdenes misteriosas, personas con capacidad de entrar sus brazos en aceite hirviendo sin quemarse" (p. 31).

De igual manera, el autor valiéndose del personaje de MacKandal introduce elementos sobrenaturales, atribuyéndole a este un conjunto de poderes "capaz de transformarse en todo tipo de ser viviente y humano muerto" (p. 44). De igual modo, presenta "niños que nacen con la mitad humana y mitad animal" (p. 44). En lo que se refiere a la parte estética, esta se puede apreciar a lo largo del desarrollo de la novela a través del retrato de los personajes y la descripción de los paisajes.

En escenario propio de la lengua española, la novela representa una rica fuente para trabajar los aspectos funcionales y estructurales de la lengua, igualmente que los aspectos y características propias de la narración, así como los aspectos lexicales. En este orden, Porrata (2002) destaca: "A partir de la publicación de El reino de este mundo empezamos a encontrar (...) una preocupación por el lenguaje: arcaísmos, pastiches y parodias" (p.90). Así pues, encontramos durante el desarrollo de la novela un uso de la lengua bastante fluido al hacer confluir en ella una gran diversidad cultural por medio de la palabra. Encontramos así la palabra "batea" (p.26) la cual proviene del árabe hispánico batía. Así también trae al escenario la palabra "mandinga". (p. 30) de origen africano y la palabra "husmear". (p. 30) con sus orígenes en el latín. Es notable también la combinación de los arcaísmos con palabras modernas y cuidadosamente seleccionadas, tal como expone Porrata (2002) al expresar que: "El discurso del narrador, las palabras rebuscadas, es antagónico a la fingida espontaneidad y oralidad. Sobresale en el discurso escrito, lo leído y premeditado, lo corregido y repensado" (p. 91). La riqueza lingüística evidenciada a la luz de estas citas y ejemplos ofrecen un escenario ideal para el estudio de la diversidad de la lengua, origen y evolución.

Respecto a los elementos estructurales de la lengua, la novela en cuestión ofrece una gran 
oportunidad para su análisis. En el caso específico de las categorías gramaticales, destaca la fluidez de los adjetivos empleados en sus descripciones, los cuales, además de cumplir una función estética, permiten al lector apreciar con mayor nitidez los escenarios en los que se desarrolla la novela y acercarse a los personajes física y emocionalmente. En este contexto, explica Porrata (2002), "Su característica principal, además de la exactitud es la sensorialidad. Lujosa la manera como se las arregla para que la historia parezca entrarle al lector por todos los sentidos: la vista, el oído, el olfato, el gusto, el tacto" (p. 92)

De igual manera, como parte de la metodología de estudio de la novela histórica, además de la identificación y agrupación de los saberes implícitos en ella, de los cuales se ha dado cuenta anteriormente, se procedió a diseñar una secuencia didáctica de actividades de enseñanza y aprendizajes, que van desde la conceptualización hasta la elaboración de productos tangibles (medibles) que dan cuenta del progreso de los estudiantes y del desarrollo de competencias fundamentales, como la resolución de problemas y el desarrollo del pensamiento crítico.

Estas actividades diseñadas para el estudio de la novela histórica están dirigidas a la construcción de conocimientos interdisciplinares, integrando tanto la lengua española, la literatura y las ciencias sociales como las ramas implicadas en este género. Dentro de estas actividades figuran: elaboración de cuadros comparativos de los elementos históricos, religiosos, culturales, políticos y económicos de la época en la que se sitúa la acción de la novela histórica abordada en relación con el ahora. Así también, se incluye el análisis de la actuación de los personajes en relación con los valores éticos y morales y su incidencia en el colectivo social. Se diseñaron mesas de diálogos para propiciar la reflexión, informes de lectura, paneles y debates en los que se fortalecieron las competencias comunicativas. Igualmente, se integraron actividades orientadas a la identificación y caracterización de la novela histórica, así como su superestructura. Para la evaluación del progreso de los estudiantes evidenciado a través de la realización de cada una de las actividades, se utilizaron rúbricas y listas de cotejo.

\section{Conclusión}

El aporte de este ensayo consiste en proponer la novela histórica como herramienta didáctica en la construcción de conocimientos interdisciplinares más que destacar su importancia y analizar su contenido como se ha hecho en la mayoría de los trabajos relacionados con la novela histórica, para lo cual hemos aportado ejemplos utilizados para explotar su contenido desde las áreas convergentes en ella. Esta posibilidad que ofrece la novela histórica en la construcción de conocimientos interdisciplinares desde nuestro enfoque es vista simultáneamente como una oportunidad para los docentes del ciclo de Estudios Generales en la que pueden establecer alianzas pedagógicas a través de sus planes de clases de cara al fortalecimiento de las competencias genéricas que se pretenden desarrollar.

De igual forma, el enfoque interdisciplinar con el que se ha trabajado la novela histórica hace posible la toma de consciencia acerca de los límites de la disciplinariedad, la riqueza y afinidad de las áreas del conocimiento partiendo de sus puntos de contacto reflejados en la interdisciplinariedad. Cabe destacar que se ha tomado la novela histórica para evidenciar su potencial educativo en el ámbito interdisciplinar, pero también existen otros géneros literarios que son igualmente valiosos para los fines y propósitos de los Estudios Generales ligados a la interdisciplinariedad.

La aplicación de este enfoque interdisciplinar para el estudio de la novela histórica a la luz de la experiencia en aula les permite a los estudiantes ampliar su mirada acerca de los acontecimientos históricos desvelados, en este caso, la revolución haitiana. Así como también hemos podido comprobar el desarrollo de su pensamiento crítico en relación con la ética y la construcción de ciudadanía y democracia, vistos estos componentes a partir del análisis de los modelos de actuación de los personajes que representan el poder político, social y económico en el hecho novelado.

A la luz de la novela analizada "El reino de este mundo" tomada como ejemplo para evidenciar el potencial educativo del subgénero propuesto en el ámbito de los Estudios Generales, se pudo observar la amplia gama de conocimientos 
implícitos en ella, ya que, además de acercar al estudiante a uno de los acontecimientos más importantes de América latina, le permitió también conocer elementos propios de la cultura, como la organización social predominante reflejada a través las categorías sociales amos y siervos. De igual manera, pudieron disfrutar del goce estético que emana de la lengua y la literatura articulados en los recursos literarios y estilísticos empleados por el autor, así también, la apreciación de los elementos ficcionales incorporados en la trama a través de la figura de Mackandal y el papel que juega en la construcción de lo real maravilloso.

En síntesis, el uso adecuado de los recursos didácticos que ofrece la literatura y, en el caso específico de este estudio, las novelas históricas, constituyen una herramienta de gran valía para provocar la reflexión de los estudiantes en relación con los sucesos históricos que han marcado la humanidad. Por lo que el análisis de una novela histórica puede convertirse en una herramienta para el desarrollo del pensamiento crítico por medio de la reflexión y la comparación del contenido histórico de la novela, en relación con fuentes puramente históricas, lo cual a su vez promueve el amor por la investigación.

Finalmente, creemos que la asociación de las áreas convergentes en la novela histórica contribuye con la visión holística e integral que se pretende desarrollar en los estudiantes a través de los Estudios Generales, por lo que se plantea la evidente necesidad de que los docentes de las diferentes disciplinas del ciclo de Estudios Generales unan esfuerzos y creen una especie de comunidad en la que, además de potencializar las habilidades de los alumnos por medio de la interdisciplinariedad de las áreas y el trabajo conjunto, puedan reflexionar sobre sus avances y desaciertos, repensar y rediseñar su práctica docente como un grupo de profesionales que persiguen metas comunes y metas individuales que se pueden fortalecer a partir del beneficio que ofrece la reflexión entre pares y la socialización de experiencias.

\section{Referencias bibliográficas}

Borja Orozco, M., Alonso Galeano, A. y Ferrer Franco, Y. (2010). Los conceptos de literatura infantil y juvenil, su periodización y canon como problemas de la literatura colombiana. Estudios de Literatura Colombiana Num. 27 (2010) julio-diciembre

Carpentier, A. (1972). El reino de este mundo. Chile: Editorial Universitaria, S.A. Inscripción No. 32,254. http://www.unl.edu.ar/ingreso/ cursos/wp-content/uploads/2018/11/1-unidad-1Modif-2.pdf. (s.f.).

Las Ciencias Sociales, s. p.-U. (2018). http://www. unl.edu.car http://www.unl.edu.ar/ingreso/ cursos/wp-content/uploads/2018/11/1-unidad-1Modif-2.pdf

Mata, C. (1995). La novela histórica: teoría y comentario (Vol. Anejos de Rilce). (I. A. Kurt Spang, Arellano y Mata Eds.) Universidad de Navarra.

Nava, E. M. (octubre/ diciembre de 2014). La Novela Histórica como Apoyo para la Enseñanza de la Historia de México. Dialnet (ISSN14056313), 57/67.

Nussbaum, M. C. (2010). Sin fines de lucro. España: Romanyá Valls S.A.

Porrata, F. E. (2002). Relectura del discurso novomundista de Alejo Carpentier y Abel Posse en el contexto de la nueva novela histórica. Florida International University. https://core.ac.uk/download/pdf/46950124. pdf

PUCMM (2016). Núcleo del plan de estudio de los estudios generales

Reverte, A. P. (29 de octubre de 2015). El valor educativo de la literatura. El País.

Ruiz, S. M. (2008). La novela Histórica como recurso didáctico para las Ciencias Sociales. Ministerio de Educación Cultura y Deporte, Subdirección general de información y Publicaciones. 